Provided for non-commercial research and education use. Not for reproduction, distribution or commercial use.

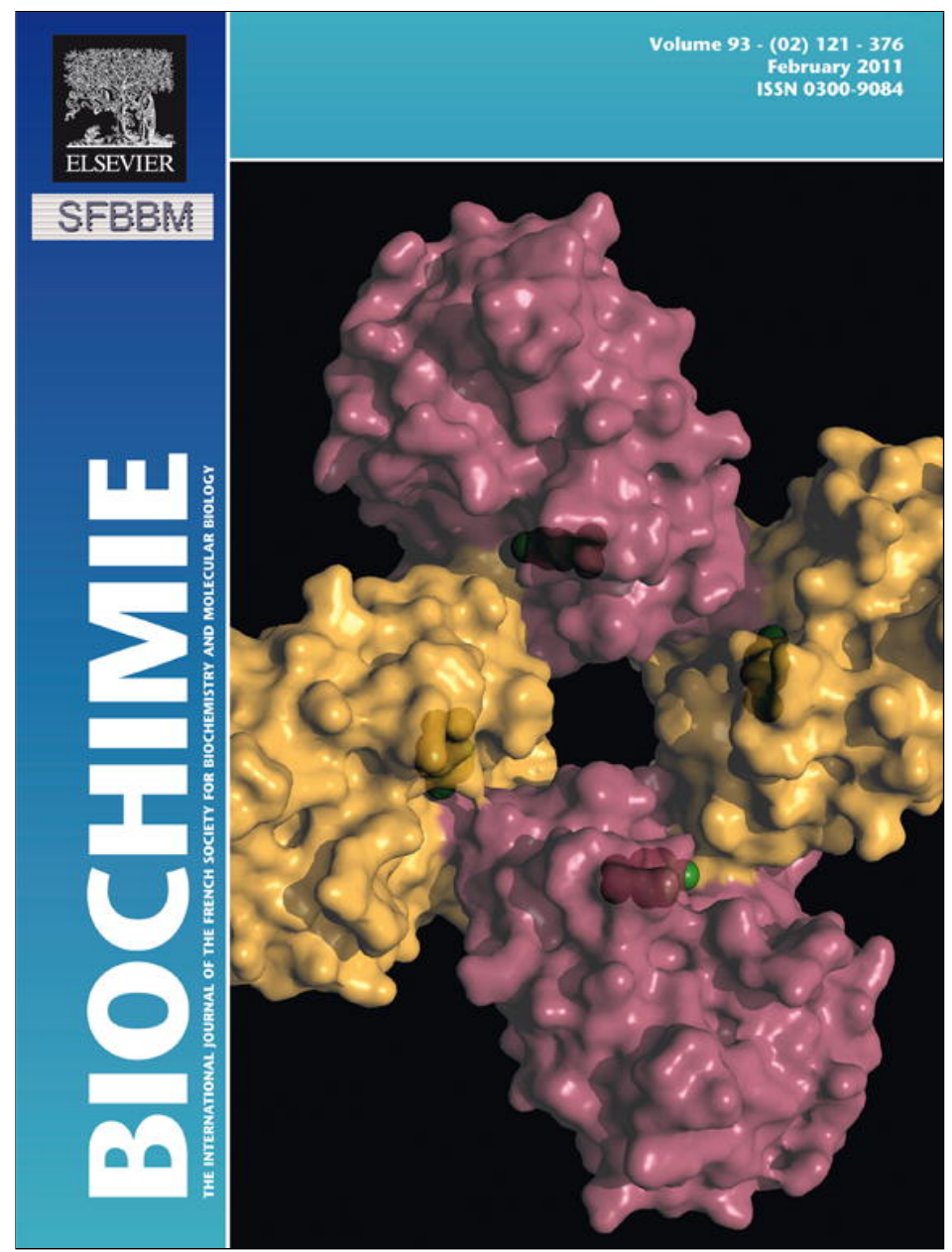

This article appeared in a journal published by Elsevier. The attached copy is furnished to the author for internal non-commercial research and education use, including for instruction at the authors institution and sharing with colleagues.

Other uses, including reproduction and distribution, or selling or licensing copies, or posting to personal, institutional or third party websites are prohibited.

In most cases authors are permitted to post their version of the article (e.g. in Word or Tex form) to their personal website or institutional repository. Authors requiring further information regarding Elsevier's archiving and manuscript policies are encouraged to visit:

http://www.elsevier.com/copyright 
Research paper

\title{
Redox regulation of UDP-glucose pyrophosphorylase from Entamoeba histolytica
}

\author{
Lucila I. Martínez ${ }^{\text {a }}$, Claudia V. Piattoni ${ }^{\text {a }}$, Sergio A. Garay ${ }^{\mathrm{b}}$, Daniel E. Rodrígues ${ }^{\mathrm{b}}$, \\ Sergio A. Guerrero ${ }^{a}$, Alberto A. Iglesias ${ }^{a, *}$
}

a Instituto de Agrobiotecnología del Litoral (UNL-CONICET), Universidad Nacional del Litoral, Ciudad Universitaria-Paraje "El Pozo", CC242, S3000ZAA Santa Fe, Argentina

b Departamento de Física. Facultad de Bioquímica y Ciencias Biológicas, Universidad Nacional del Litoral, Ciudad Universitaria-Paraje “El Pozo”, CC242, S3000ZAA Santa Fe, Argentina

\section{A R T I C L E I N F O}

\section{Article history:}

Received 13 April 2010

Accepted 22 September 2010

Available online 1 October 2010

\section{Keywords:}

UDP-glucose pyrophosphorylase

Entamoeba histolytica

Redox

Carbohydrate metabolism

Thiol

\begin{abstract}
A B S T R A C T
Amoebiasis is an intestinal infection caused by the human pathogen Entamoeba histolytica and representing the third leading cause of death by parasites in the world. Host-parasite interactions mainly involve anchored glycoconjugates localized in the surface of the parasitic cell. In protozoa, synthesis of structural oligo- and polysaccharides occurs via UDP-glucose, generated in a reaction catalyzed by UDPglucose pyrophosphorylase. We report the molecular cloning of the gene coding for this enzyme from genomic DNA of E. histolytica and its recombinant expression in Escherichia coli cells. The purified enzyme was kinetically characterized, catalyzing UDP-glucose synthesis and pyrophosphorolysis with $V_{\text {max }}$ values of $95 \mathrm{U} / \mathrm{mg}$ and $3 \mathrm{U} / \mathrm{mg}$, respectively, and affinity for substrates comparable to those found for the enzyme from other sources. Enzyme activity was affected by redox modification of thiol groups. Different oxidants, including diamide, hydrogen peroxide and sodium nitroprusside inactivated the enzyme. The process was completely reverted by reducing agents, mainly cysteine, dithiothreitol, and thioredoxin. Characterization of the enzyme mutants C94S, C108S, C191S, C354S, C378S, C108/378S, M106S and M106C supported a molecular mechanism for the redox regulation. Molecular modeling confirmed the role of specific cysteine and methionine residues as targets for redox modification in the entamoebic enzyme. Our results suggest that UDP-glucose pyrophosphorylase is a regulated enzyme in E. histolytica. Interestingly, results strongly agree with the occurrence of a physiological redox mechanism modulating enzyme activity, which would critically affect carbohydrate metabolism in the protozoon.
\end{abstract}

(c) 2010 Elsevier Masson SAS. All rights reserved.

\section{Introduction}

Entamoeba histolytica is a parasite responsible for intestinal amoebiasis and amoebic liver abscess, exclusively in humans [1]. Many people, mainly in developing countries, chronically suffer amoebiasis; this disease having high morbidity and mortality factors in the poor population and thus causing substantial economic burden on public health. The parasitic protozoon has a simple life cycle: an infectious cystic form, which is ingested via contaminated water or food; and an invasive amoeboid trophozoite stage. Trophozoites are the motile forms with capacity to colonize and occasionally invade the colonic mucosa [2]. The cell surface of $E$. histolytica is decisive for recognition and killing of target host cells, with several molecules clearly identified for their

Abbreviations: DTT, dithiothreitol; GPI, glycosylphosphatidylinositol; RNS, nitrogen reactive species; ROS, oxygen reactive species; SNP, sodium nitroprusside; TRX, thioredoxin; TXN, tryparedoxin; UDP-GlcPPase, UDP-glucose pyrophosphorylase; UDP-Gal, UDP-galactose; UDP-Glc, UDP-glucose.

* Corresponding author. Tel./fax: +54 342 4575209x217.

E-mail address: iglesias@fbcb.unl.edu.ar (A.A. Iglesias). involvement in amoebic pathogenesis. E. histolytica displays a class of highly polymorphic glycosylphosphatidylinositol (GPI)anchored cell-surface glycoconjugates, originally referred as lipophosphoglycans (LPGs). Antibodies against LPGs inhibit adhesion of trophozoites to mammalian cells, indicating that the antigen may be involved in host-parasite interactions $[1,2]$.

The detailed structure of the glycoconjugate present in LPGs has been elucidated. It is now clear its proteophosphoglycan (PPG) structure, containing a novel GPI-anchor (Gal1Man2GlcN-myo inositol) linked to a highly acidic polypeptide [3]. Less is known about specific biosynthetic pathways in E. histolytica rendering PPG or any other complex glycoconjugate. Production of structural oligoand polysaccharides occurs via UDP-Glc in different organisms $[4,5]$. The sugar-nucleotide is synthesized from glucose-1-phosphate (Glc1P) and UTP in a reaction catalyzed by UDP-Glc pyrophosphorylase (EC 2.7.7.9; UDP-GlcPPase) [6-8]. In this context, the enzyme would play a relevant role in $E$. histolytica, catalyzing the step initiating metabolic routes leading to the formation of a variety of carbohydrates, which are critical for the protozoan survival and pathogenesis. Despite this relevance, UDP-GlcPPase from this organism has received very scarce attention and the absence of 
information about its kinetic and structural properties restricts the understanding of carbohydrate metabolism in the parasite. Herein, we report the molecular cloning, expression and characterization of UDP-GlcPPase from E. histolytica. Remarkably, results show that the enzyme activity can be regulated by redox modification exerted by key metabolites of the intracellular environment, which envision the occurrence of a physiological mechanism for postraductional protein modification in the parasite.

\section{Materials and methods}

\subsection{Bacterial strains and plasmids}

Escherichia coli Top 10 and E. coli BL21 (DE3) cells were utilized in routine molecular biology assays (plasmid construction and protein expression). The vector $\mathrm{pCR}^{\circledR} 4 \mathrm{Blunt}^{\mathrm{TOOPO}}{ }^{\circledR}$ was selected for cloning and sequencing purposes, whereas pRSET-B was the plasmid utilized for protein expression all the specified bacteria strains and plasmids were from Invitrogen. Cells and genomic DNA from E. histolytica HM-1:IMSS were kindly provided by Dr. Hugo Lujan (Universidad Católica de Córdoba-CONICET, Argentina). DNA manipulation, E. coli cultures, and transformation procedures were performed according to standard protocols [9].

\subsection{DNA techniques and plasmids construction}

Specific oligonucleotide primers were designed after information available in the NCBI database (http://www.ncbi.nlm.nih.gov). The forward primer (Fo) contained a BamHI site and overlapped the $5^{\prime}$-end of the coding sequence, whereas the reverse primer $(R e)$ overlapped the $3^{\prime}$ end of the coding sequence and contained a HindIII site (see Table 1). The gene was amplified from E. histolytica genomic DNA by PCR techniques $\left(95^{\circ} \mathrm{C}, 1 \mathrm{~min}\right.$; $95^{\circ} \mathrm{C}, 1 \mathrm{~min}$; $55^{\circ} \mathrm{C}, 1 \mathrm{~min} ; 72{ }^{\circ} \mathrm{C}, 2 \mathrm{~min} ; 30$ cycles; $72{ }^{\circ} \mathrm{C}, 5 \mathrm{~min}$ ). PCR amplification was performed using $1.25 \mathrm{U}$ of Pfu DNA polymerase (Fermentas), $1 \mu \mathrm{g}$ of parasite genomic DNA, $1 \mu \mathrm{M}$ of each synthetic oligonucleotides, and $50 \mu \mathrm{M}$ of each deoxynucleoside triphosphate in a buffer with $2 \mathrm{mM} \mathrm{MgCl}$. The PCR product was subsequently purified and ligated into the $\mathrm{PCR}^{\circledR} 4 \mathrm{Blunt}-\mathrm{TOPO}^{\circledR}$ to facilitate further works. Fidelity and correctness of the gene was confirmed by complete sequencing.

\subsection{Expression of recombinant protein}

Standard preparation of the plasmid $\left[\mathrm{pCR}^{\circledR} 4 \mathrm{Blunt}-\mathrm{TOPO}^{\circledR} /\right.$ EhiUDP-GlcPPase] was digested with BamHI and HindIII enzymes. The fragment encoding EhiUDP-GlcPPase was cloned into a pRSET$B$ expression vector between the same restriction sites. Cells of $E$. coli BL21 (DE3) transformed with the recombinant plasmid [pRSET$\mathrm{B} /$ EhiUDP-GlcPPase] were grown in YT2X medium (16 $\mathrm{g} / \mathrm{l}$ of yeast extract, $10 \mathrm{~g} / \mathrm{l}$ of peptone, $5 \mathrm{~g} / \mathrm{l}$ of $\mathrm{NaCl}$ ) with $100 \mu \mathrm{g} / \mathrm{ml}$ of ampicillin, at $28^{\circ} \mathrm{C}$ and shaking at $180 \mathrm{rpm}$. When the cell culture reached an $\mathrm{OD}_{600}$ of $0.6-0.8$, overexpression of the recombinant EhiUDPGlcPPase was induced with $1 \mathrm{mM}$ IPTG during $20 \mathrm{~h}$. After centrifugation at $5000 \mathrm{~g}$ for $10 \mathrm{~min}$, the pellet was recovered and resuspended in buffer $\mathrm{A}(20 \mathrm{mM}$ Tris- $\mathrm{HCl}, \mathrm{pH} 8.0,300 \mathrm{mM} \mathrm{NaCl}$ and $10 \mathrm{mM}$ imidazole). Cells were disrupted by sonication and centrifuged at $10000 \mathrm{~g}$ for $20 \mathrm{~min}$. Soluble and insoluble fractions in crude extracts were analyzed by SDS/PAGE.

\section{4. $\mathrm{Ni}^{2+}$-chelating chromatography}

The soluble fraction was loaded (flow rate of $2 \mathrm{ml} / \mathrm{min}$ ) onto a $\mathrm{Ni}^{2+}$-IDA-agarose resin (Invitrogen) previously equilibrated with buffer A. After washing with 10 bed volumes of buffer $A$, the recombinant protein was eluted with buffer A containing $300 \mathrm{mM}$ imidazole. Fractions containing EhiUDP-GlcPPase activity were pooled and stored at $-80^{\circ} \mathrm{C}$ in the same buffer supplemented with $0.1 \mathrm{mM}$ EDTA, $1 \mathrm{mM}$ dithiothreitol (DTT) and 7\% (v/v) glycerol.

\subsection{Protein methods and molecular mass determination}

Protein contents were determined using the method of Bradford [10], with BSA as a standard. Purified protein and cell-free extracts were analysed by SDS/PAGE [11] using a Mini-PROTEANII (Bio-Rad) apparatus. The final polyacrylamide monomer concentration was $15 \%(\mathrm{w} / \mathrm{v})$ for the resolving gel and $4 \%(\mathrm{w} / \mathrm{v})$ for the stacking gel. Coomassie Brilliant blue staining was used to visualize proteins in the gel. Molecular mass standards used in SDS/PAGE were from GE-Healthcare: rabbit muscle phosphorylase b (97 kDa), bovine serum albumin (66 kDa), chicken egg white ovalbumin (45 kDa), bovine erythrocyte carbonic anhydrase (30 kDa), soybean trypsin inhibitor (20.1 kDa), and bovine milk $\alpha$-lactalbumin (14.4 kDa).

Detection of specific proteins in crude extracts from $E$. histolytica trophozoites after Western blotting was performed following standard techniques [9]. Proteins in SDS/PAGE gels were blotted onto PVDF membranes. The membrane was blocked overnight at $4{ }^{\circ} \mathrm{C}$, subsequently incubated with the primary antibody raised against Trypanosoma brucei UDP-GlcPPase (pure enzyme was obtained recombinantly and antibodies produced according to [12]) at room temperature for $1 \mathrm{~h}$, and then incubated with a HRPconjugated anti-rabbit secondary antibody for $1 \mathrm{~h}$. Detection was carried out with 3,3'-diaminobenzidine and hydrogen peroxide (Sigma-Aldrich) in $50 \mathrm{mM}$ Tris- $\mathrm{HCl}, \mathrm{pH} 8.0,150 \mathrm{mM} \mathrm{NaCl}$.

The molecular mass of EhiUDP-GlcPPase was determined by size exclusion chromatography carried out on a Superdex 200 10/30 HR column (GE-Healthcare) using a standard method. The following protein standards were utilized: carbonic anhydrase (29 kDa), ovoalbumin (45 $\mathrm{kDa})$, bovine serum albumin (66 kDa) and rabbit IgG (140 kDa). Samples were chromatographed with elution buffer (0.1 $\mathrm{M} \mathrm{NaCl}, 0.1 \mathrm{mM}$ EDTA, $2 \%$ glycerol and $25 \mathrm{mM}$ Tris- $\mathrm{HCl}, \mathrm{pH} 7.5$ ) at a flow rate of $0.5 \mathrm{ml} / \mathrm{min}$.

Table 1

Primers used for ehiugp gene. Forward and reverse oligonucleotides for wild type gene amplification (restriction sites underlined) and site-directed mutagenesis (in bold are the bases that were changed).

\begin{tabular}{|c|c|c|}
\hline Primer & Oligonucleotide Fo & Oligonucleotide $R e$ \\
\hline Wild type & 5'-GGATCCCATGGAACTCAAAATTAATCA-3' & 5'-AAGCTTTTAGAATGTTAATGTAGTAT-3' \\
\hline C94S & 5'-TTAAAGAAAACTTCTATTATTAAG-3' & 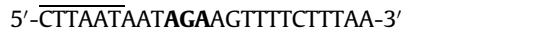 \\
\hline C108S & 5'-CATCTATGGGATCTACTGGTCCAAAAG-3' & 5'-CTTTTTGGACCAGTAGATCCCATAGATG-3' \\
\hline C191S & 5'-CTCTTCTTCCTGTATCTACTGAATTAAATGG-3' & 5'-CCATTTAATTCAGTAGATACAGGAAGAAGAG-3' \\
\hline C354S & 5'-GAAATTGCAGTTGGATCTGCAGTAAGTGCATTT- $3^{\prime}$ & 5'-AAATGCACTTACTGCAGATCCAACTGCAATTTC- $3^{\prime}$ \\
\hline C378S & 5'-CCAGTAAAAGCATCTAATGATTTATTT-3' & 5'-AAATAAATCATTAGATGCTTTTACTGG-3' \\
\hline M106C & 5'-GGATTAGGAACATCTTGTGGATGTACTGGT-3' & 5'-ACCAGTACATCCACAAGATGTTCCTAATCC-3' \\
\hline M106S & 5'-GGATTAGGAACATCTTCGGGATGTACTGGT-3' & 5'-ACCAGTACATCC̄CGAAGATGTTCCTAATCC-3' \\
\hline
\end{tabular}




\subsection{Enzyme activity assays}

Activity of UDP-GlcPPase was assayed in both directions of catalysis, pyrophosphorolysis (assay A) or synthesis (assays B) of UDP-Glc. All the assays were performed at $37{ }^{\circ} \mathrm{C}$ and $\mathrm{pH}$ 8.0. One unit $(U)$ of activity is the amount of enzyme catalyzing the formation of $1 \mu \mathrm{mol}$ of product (either UTP, or UDP-Glc, as determined by assays A or B, respectively) per min under the specified assay conditions.

\subsubsection{Assay A}

Formation of $\left[{ }^{32} \mathrm{P}\right] \mathrm{UTP}$ from $\left[{ }^{32} \mathrm{P}\right] \mathrm{PPi}$, in the direction of pyrophosphorolysis of UDP-Glc was determined by the method previously described for pyrophosphorylases [13]. The reaction was carried out for $10 \mathrm{~min}$ in a mixture containing (unless otherwise indicated) $100 \mathrm{mM}$ MOPS, $5 \mathrm{mM} \mathrm{MgCl}, 1 \mathrm{mM}\left[{ }^{32} \mathrm{P}\right] \mathrm{PPi}$ (1500-2500 cpm/nmol), $1 \mathrm{mM}$ UDP-Glc, $4 \mathrm{mM} \mathrm{NaF}$, and $0.05 \mathrm{mg} /$ $\mathrm{ml}$ BSA, plus enzyme in a total volume of $250 \mu \mathrm{l}$. After $10 \mathrm{~min}$ incubation, the reaction was stopped by the addition of $3 \mathrm{ml}$ cold $5 \%$ $(\mathrm{w} / \mathrm{v})$ trichloroacetic acid and $\left.{ }^{32} \mathrm{P}\right] \mathrm{UTP}$ formed was bound to activated carbon (15\% Norite A in $100 \mathrm{mM} \mathrm{PPi}$ ). After washing twice the UTP-bound carbon with $3 \mathrm{ml}$ each 5\% (w/v) trichloroacetic acid, UTP was hydrolyzed by addition of $\mathrm{HCl}$ to $1 \mathrm{M}$ and boiling for $10 \mathrm{~min}$ in a water-bath. Released radioactive Pi was measured in a scintillation counter.

\subsubsection{Assay $B$}

Synthesis of UDP-Glc and PPi from Glc1P and UTP was followed by measuring Pi generated from PPi using the high sensitivity, colorimetric method developed by Fusari et al. [14]. The reaction was carried out for $10 \mathrm{~min}$ in a mixture that contained (unless otherwise specified) $100 \mathrm{mM}$ MOPS, $5 \mathrm{mM} \mathrm{MgCl} 2,1 \mathrm{mM} \mathrm{Glc1P}$, $1 \mathrm{mM}$ UTP, $0.015 \mathrm{U} / \mu \mathrm{l}$ pyrophosphatase, and $0.05 \mathrm{mg} / \mathrm{ml} \mathrm{BSA}$, plus enzyme in a total volume of $50 \mu \mathrm{l}$. The reaction was terminated with the addition of Malachite-green color reagent, and read at $650 \mathrm{~nm}$ with a Multiskan Ascent 384-microplate reader (Thermo Electron).

\subsection{Kinetic characterization}

Kinetic data were plotted as specific activity $\left(\mathrm{U} \cdot \mathrm{mg}^{-1}\right)$ versus substrate concentration. EhiUDP-GlcPPase utilized in the kinetic studies was the fully reduced enzyme, maintained in the presence of $1 \mathrm{mM}$ DTT and checked for full activity. Kinetic constants were acquired by fitting the data to the Hill equation with a non-linear least-squares formula using the program Origin ${ }^{\mathrm{TM}}$ 7.0. Hill plots were used to calculate Hill coefficient and the kinetic constants that correspond to maximal velocity $\left(V_{\max }\right)$ as well as the substrate concentrations giving $50 \%$ of the maximal velocity $\left(S_{0.5}\right)$. Kinetic constants are means of at least three independent sets of data that were reproducible within $\pm 10 \%$.

\subsection{Site-directed mutagenesis}

Mutant enzymes in cysteine (C94S, C108S, C191S, C354S, C378S, and $\mathrm{C} 108 / 378 \mathrm{~S}$ ) and methionine (M106S, and M106C) residues were constructed by using the QuikChange ${ }^{\mathrm{TM}}$ (Stratagene) sitedirected mutagenesis kit. The sequence of each mutant was verified by double strand sequencing. Oligonucleotides used for mutagenesis are shown in Table 1.

\subsection{Redox modification}

Experimental points determined in redox modification studies are means of at least three measurements and they were reproducible within $\pm 10 \%$.

\subsubsection{Oxidation assay}

Purified EhiUDP-GlcPPase was incubated, at $25^{\circ} \mathrm{C}$ in the presence of different concentrations either of diamide, hydrogen peroxide, or sodium nitroprusside (SNP). The incubation medium contained $50 \mathrm{mM}$ buffer MOPS (pH 8.0). The incubation with SNP was carried out with direct environment light to induce photolytic decomposition of the reagent and generate nitric oxide and others reactive nitrogen species [15]. After different incubation times, aliquots were withdrawn and assayed for UDP-GlcPPase activity using the colorimetric method [14]. Identical treatments were performed for the mutant enzymes.

\subsubsection{Reduction assay}

EhiUDP-GlcPPase was first oxidized as specified above. Then, the oxidant was removed by washing with the same buffer and using Microcom columns (Millipore). The oxidized enzyme was incubated with the different reducing agents (see details below). After 30 min incubation, aliquots were withdrawn from the incubation media and assayed for UDP-GlcPPase activity using the colorimetric method [14] Reducing agents utilized were: E. histolytica thioredoxin 8 (EhiTRX8) $(0-5 \mu \mathrm{M})$, E. histolytica thioredoxin 41 (EhiTRX41) $(0-5 \mu \mathrm{M})$, Trypanosoma brucei thioredoxin (TbrTRX) $(0-5 \mu \mathrm{M})$, Trypanosoma cruzi tryparedoxin (TcrTXN) $(0-5 \mu \mathrm{M})$, DTT $(0-120 \mu \mathrm{M})$ and L-cysteine hydrochloride monohydrate (Sigma-Aldrich) $(0-200 \mu \mathrm{M})$. The thiol proteins utilized as reducing agents were purified according to protocols previously specified by Arias et al., Reckenfelderbäumer et al. and Wilkinson et al. respectively [16-19]. Previous to use, all the thiol proteins had been exhaustively reduced by incubation during $30 \mathrm{~min}$ at $25^{\circ} \mathrm{C}$ in a medium containing $50 \mathrm{mM}$ buffer MOPS pH 8.0, $0.2 \mathrm{mg} / \mathrm{m} \mathrm{BSA}$, and $0.5 \mathrm{mM}$ DTT.

\subsubsection{Determination of intramolecular disulphide bridge}

Enzyme was oxidized with either $0.5 \mathrm{mM}$ diamide, $1 \mathrm{mM}$ hydrogen peroxide, or $5 \mathrm{mM}$ SNP under the conditions specified in 2.9.1. After oxidation, each sample derived in two as they received no further treatment or were incubated with $25 \mathrm{mM}$ DTT for $10 \mathrm{~min}$. Oxidized and reduced samples were analyzed by SDS/PAGE using non-reducing or reducing sample buffer, respectively.

\subsection{Molecular modeling}

The search for suitable template models was made with the template identification tool of the Swiss Model Workspace [20]. Sequences of the selected templates were aligned using the program STAMP [21], which is available in VMD software. Finally our target was aligned with the previously obtained using Clustal X 1.81 [22]. Special care was taken to avoid any gap inside of secondary structure elements. Based on the last alignment, 25 homology models of EhiUDP-GlcPPase were obtained using the software Modeller 8v2 [23]. The best model was established taken into account both, the higher global score of verify3D [24] and also the higher DOPE1 potential values. The program PROCHECK, validating structures, was run to check the stereochemistry quality of the best model.

\section{Results}

\subsection{Expression and properties of recombinant EhiUDP-GlcPPase}

The identification of a nucleotide sequence (GeneID: 3407065 , actualized under gi: 67474001) encoding a putative UTP-glucose-1phosphate uridylyltransferase in the NCBI database prompted us to perform the molecular cloning of the full-length gene (ehiugp). The gene was amplified from genomic DNA and its identity was confirmed by DNA sequencing. This gene (1,446 bp in length) is predicted to encode a 481 amino acids protein (EhiUDP-GlcPPase) 


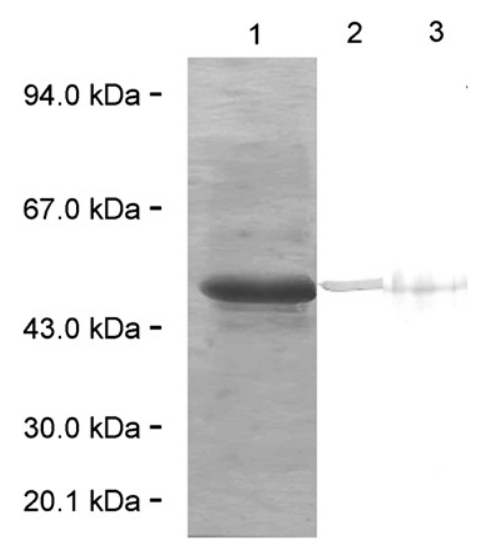

Fig. 1. Electrophoretic analysis of EhiUDP-GlcPPase. Lane 1, SDS/PAGE of the recombinant EhiUDP-GlcPPase $(2 \mu \mathrm{g})$ stained with Coomassie blue. Lane 2, Western blotting of the above gel revealed with antiserum raised against UDP-GIcPPase from $T$. brucei. Lane 3, Western blotting of a crude extract from E. histolytica $\left(5 \times 10^{5}\right.$ cells $)$ resolved by SDS/PAGE and revealed with rabbit polyclonal anti-TbrUDP-GlcPPase.

with a calculated pI of 6.2. To further seek the protein functionality, the gene encoding EhiUDP-GlcPPase was cloned into the prokaryotic expression vector pRSET-B. Expression in E. coli BL21 (DE3) rendered a recombinant protein produced as a polypeptide tagged with histidine residues at the $\mathrm{N}$-terminus. The soluble fraction was chromatographically purified onto a $\mathrm{Ni}^{2+}$-affinity resin, to obtain the protein with purity higher than $98 \%$, as judged by SDS/PAGE analysis (Fig. 1). The molecular mass thus revealed for the protein fully agrees with the expected size $(54.8 \mathrm{kDa})$, deduced from its amino acids sequence, plus ca. $3 \mathrm{kDa}$ (corresponding to residues in the His-tag). This size for the protein subunit is also similar to those from other UDP-GlcPPases found in eukaryotes [7,25]. Western blot analysis determined that the purified recombinant enzyme was recognized by antibodies raised against the UDP-GlcPPase from T. brucei (Fig. 1). The latter specific labeling was useful to evidence the occurrence of UDP-GlcPPase in crude extracts obtained from $E$. histolytica cells (Fig. 1). The quaternary structure of the pure protein was analyzed by size exclusion chromatography (see Experimental). The recombinant enzyme eluted as a single peak with retention volume corresponding to a monomeric structure, in agreement with that reported for the enzyme from Leishmania major [7].

The recombinant protein exhibited activity in catalyzing synthesis (forward direction) and pyrophosphorolysis (reverse direction) of UDP-Glc. Table 2 shows the kinetic properties of the enzyme respect to the different substrates and the essential cofactor

Table 2

Kinetic parameters of purified EhiUDP-GlcPPase ${ }^{\mathrm{a}}$

\begin{tabular}{lccc}
\hline Substrate & $S_{0.5}(\mathrm{mM})$ & $n_{\mathrm{H}}$ & $V_{\max }(\mathrm{U} / \mathrm{mg})$ \\
\hline \multicolumn{2}{l}{ Pyrophosphorolysis direction } & & \\
UDP-Glc & 0.44 & 1.6 & 3.0 \\
PPi & $\sim 0.94$ & 1.2 & \\
$\mathrm{Mg}^{2+}$ & 0.06 & 1.0 & \\
Synthesis direction & & & \\
Glc1-P & 0.25 & 1.0 & 95.0 \\
UTP & 0.81 & 1.9 & \\
$\mathrm{Mg}^{2+}$ & 0.06 & 1.9 & \\
\hline
\end{tabular}

a Assay conditions are as specified under Materials and Methods, except that for the respective variable substrate the range of concentrations utilized were $0-10 \mathrm{mM}$ for Glc1P, $0-5 \mathrm{mM}$ for UTP, $0-10 \mathrm{mM}$ for UDP-Glc, $0-2 \mathrm{mM}$ PPi. Kinetic constants were acquired by fitting the data to the Hill equation with a non-linear least-squares formula. Kinetic constants are means of at least three independent sets of data that were reproducible within $\pm 10 \%$. Note that for the case of PPi the $S_{0.5}$ in approximate, as we only varied the substrate up to $2 \mathrm{mM}$ (levels lower than saturating), because higher concentrations produced precipitation in assay media.
$\mathrm{Mg}^{2+}$. The $V_{\max }$ for the reaction of UDP-Glc synthesis was over 30fold higher than that determined for pyrophosphorolysis, although the affinity for substrates was in the same order for both directions of catalysis (Table 2). The values of activity in the forward direction thus obtained is similar to that previously found for the enzyme from Xanthomonas spp. [6]. The reaction catalyzed by pyrophosphorylases is freely reversible in vitro, with an equilibrium close to 1 ; although differences were found respect to rates of the forward or reverse reaction [26], which could be attributed to regulation or characteristics associated with protein oligomerization [6,27]. Data in Table 2 indicate that the EhiUDP-GlcPPase exhibited lower $V_{\max }$ and affinity for substrates than the enzyme from $L$. major [5,7], Arabidopsis thaliana [28], and barley [29]; whereas kinetics for the recombinant enzyme are similar than those reported for one specific UDP-GlcPPase partially purified from E. histolytica [30], except for a lower affinity of the former for UTP. In our hands, the entamoebic enzyme was not able to utilize ATP, GTP, TTP, or mannose-1-P as substrates, these results agreeing with the previous characterization of the enzyme purified from cells of the protozoan [30]. As a whole, these results on specificity are in agreement with data previously reported for other UDP-GlcPPases [6-8], except for the use of TTP as a substrate by the enzyme from Xanthomonas spp. [6]. Moreover, the recombinant enzyme was insensitive to regulation by any allosteric effector. So far, we analyzed that the activity of EhiUDP-GlcPPase was not affected by different metabolites that have been characterized as regulators of eukaryotic ADP-GlcPPases (such as phosphoenolpyruvate, pyruvate, fructose-6P, glucose-6P, 3P-glycerate, fructose-1,6-bisphosphate, AMP, and Pi) [26].

\subsection{Redox modulation}

The primary structure of EhiUDP-GlcPPase contains five cysteine and eleven methionine residues. We analyzed if the activity of the purified recombinant enzyme could be affected by incubation with three compounds: diamide, hydrogen peroxide, and SNP, which are recognized to be able of modifying the redox status of S-containing moieties. SNP generates nitric oxide-like reactive species able to produce protein S-nitrosylation [15]. Fig. 2 show that incubation with the three oxidant agents affected the enzyme activity, by inhibiting it in a time dependent, irreversible manner. A more detailed study, varying inhibitor levels indicated that the loss of enzyme activity followed pseudo-first order kinetics; with degrees of reactivity established from inactivation constants $\left(K_{\mathrm{I}}\right)$ determined by the reciprocal of the product between time (for reaching $50 \%$ of inactivation) and reagent concentration. In this way, diamide exhibited the higher degree of reactivity, followed by hydrogen peroxide and SNP (inset of Fig. 2). Nevertheless, the three compounds are effective oxidants acting in the same order of concentration; with levels of hydrogen peroxide and SNP able to inactivate the enzyme within $10 \mathrm{~min}$ being in the $1-5 \mathrm{mM}$ range. The latter is compatible with values found in different cells for oxidizing and S-nitrosylating species [31,32]. The enzyme inactivation was partially protected by UTP, with no effect exerted by the substrate Glc1P.

Interestingly, the inhibitory effect of oxidizing agents on the activity of EhiUDP-GlcPPase could be reverted by incubation of the oxidized enzyme with different reductants (Fig. 3 ). In such a way, Fig. 3 shows that were effective for reversal the chemical agent DTT, as well as the biological effectors TcrTXN and TbrTRX. The latter has a potential physiological relevance, as redox agents found in cells at comparable concentrations $(0-5 \mu \mathrm{M})$ are able of exert recovery of the enzyme activity. Fig. 4 strongly supports this view, because agents specifically found in E. histolytica, as L-cysteine [the main low molecular mass thiol in the parasite [33], EhiTRX8 and EhiTRX41 also were effective in reverting EhiUDP-GlcPPase from an 


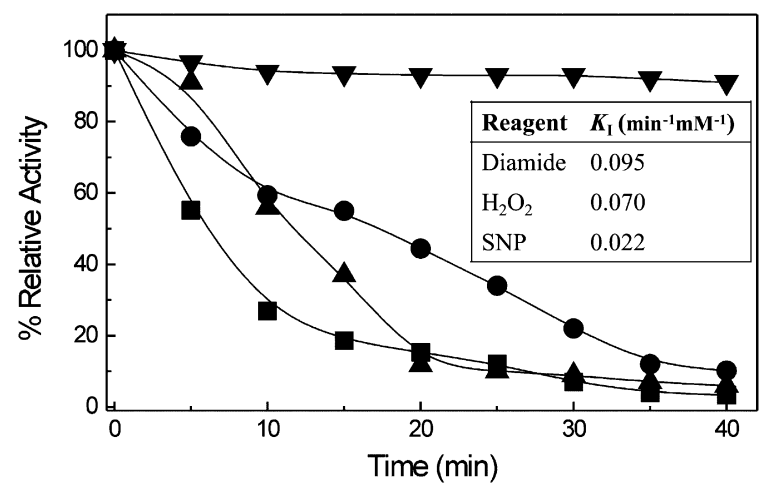

Fig. 2. Inactivation of EhiUDP-GlcPPase by different oxidants. The enzyme was incubated at $\mathrm{pH} 8.0$ and $25{ }^{\circ} \mathrm{C}$ with $0.5 \mathrm{mM}$ diamide $(\bullet), 1 \mathrm{mM}$ hydrogen peroxide $(\boldsymbol{\Lambda})$ $5 \mathrm{mM}$ of SNP ( $\boldsymbol{\nabla})$ or without further additions ( $\boldsymbol{\nabla}$ ) (control). At the specified times, aliquots were withdrawn and assayed for UDP-Glc synthesis activity. Inset: Pseudo-first order inactivation constants $\left(K_{\mathrm{I}}\right)$ from treatment of the enzymes with varying concentrations of each oxidant and determined by the reciprocal of the product between time for reaching $50 \%$ of inactivation and reagent concentration.

inactive to a fully active state. An analysis of the reduced and oxidized forms of the enzyme revealed that the former migrates faster in SDS/PAGE (Fig. 5). As illustrated by Fig. 5A, changes in the apparent motility between the two redox forms of the enzyme were found to be respectively achieved after treatment with the different redox agents causing oxidation or reduction. Additionally, as shown in Fig. $5 \mathrm{~B}$, intermediate forms of the protein were visualized in SDS/PAGE when the oxidized enzyme was reduced to different degrees with various amounts $(0-12 \mu \mathrm{M})$ of EhiTRX41. The latter agrees with a correlation between the reduction of disulfide bond and the recovery of enzyme catalytic capacity, varying from $10 \%$ residual activity (Fig. 5 A, lane 1 ) to a fully active EhiUDP-GlcPPase (Fig. 5B, lane 7). The dissimilar apparent size of the oxidized or reduced enzyme of $\sim 7 \mathrm{kDa}$ is not compatible with intersubunit crosslinking, but it suggests the formation of intramolecular disulphide bonds after oxidation. It has been reported that the covalent crosslink between cysteine residues within one polypeptide facilitates the formation of a more compact structure $[34,35]$ and this could modify the amount of SDS that binds per mass of protein, thus modifying the electrophoretic motility. These

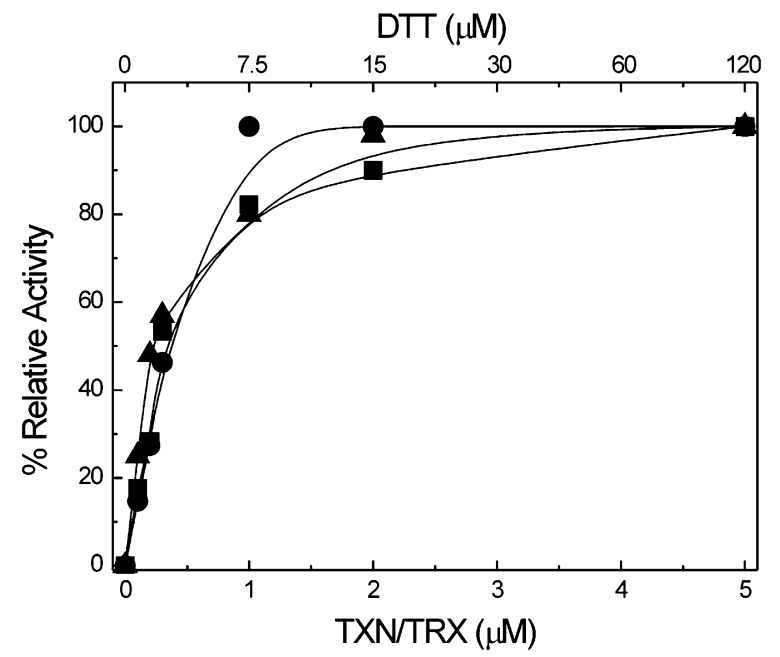

Fig. 3. Effect of reducing agents on oxidized EhiUDP-GlcPPase. Oxidized, inactive UDPGlcPPase was incubated at $\mathrm{pH} 8.0$ and $25^{\circ} \mathrm{C}$ with the specified concentrations of DTT $(\bullet), \operatorname{TbrTRX}_{\text {red }}(\boldsymbol{\square})$, or $\operatorname{TcrTXN}_{\text {red }}(\boldsymbol{\Delta})$. After $30 \mathrm{~min}$ incubation samples were assayed for UDP-Glc synthesis activity.

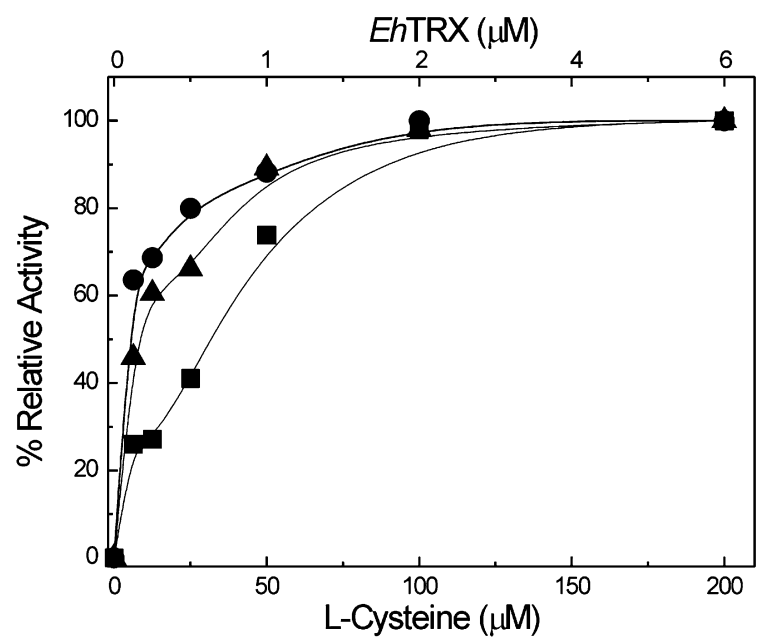

Fig. 4. Effect of L-Cysteine and EhiTRX on oxidized EhiUDP-GlcPPase. Oxidized, inactive UDP-GlcPPase was incubated at pH 8.0 and $25^{\circ} \mathrm{C}$ with the specified concentrations of L-Cysteine ( $\boldsymbol{\square})$, EhiTRX41( $\bullet$ ), or EhiTRX8 ( $\boldsymbol{\Delta})$. After 30 min samples were assayed for UDP-GlcPPase activity using the colorimetric method.

results strongly suggest a redox regulation of EhiUDP-GlcPPase, where the formation or rupture of disulphides might be a relevant mechanism. It would be important to identify the residues in the enzyme involved in such a redox modification, and homology modeling is an accurate tool to pin-point cysteines by establishing relative distances between them in the protein 3-D structure.

\subsection{Molecular modeling}

Building of the structure model of EhiUDP-GlcPPase was based in templates detailed in Table 3; derived from the known X-ray structure of the homologous enzyme from eukaryotes $[5,36,37]$. Almost all selected templates have significant number of identical residues (ca. 44\%). The UDP-GlcPPase from L. major (2oeg) has less number of identical residues (33\%) but it was incorporated to widen the diversity of folding possibility into our model. The global

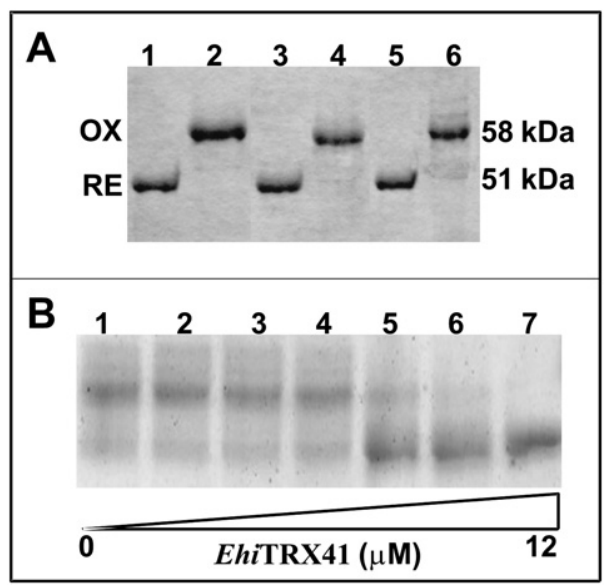

Fig. 5. Analysis of different redox states of EhiUDP-GlcPPase by SDS/PAGE. (A) Previous to electrophoresis, the purified recombinant enzyme was oxidized by 30 min treatment with $5 \mathrm{mM}$ each, diamide (lane 2), SNP (lane 4), or hydrogen peroxide (lane 6). The enzyme oxidized by the three respective oxidizing agents was later reduced again by 30 min treatment with $5 \mathrm{mM}$ DTT and analyzed by SDS/PAGE (lanes 1, 3, and 5). (B) Previous to electrophoresis the purified recombinant enzyme was oxidized by $40 \mathrm{~min}$ treatment with $0.1 \mathrm{mM}$ diamide (lane 1). The oxidized enzyme was later reduced again by 30 min treatment with $0.1 \mu \mathrm{M}$ (lane 2), $0.5 \mu \mathrm{M}$ (lane 3), $1 \mu \mathrm{M}$ (lane 4), $2 \mu \mathrm{M}$ (lane 5), $6 \mu \mathrm{M}$ (lane 6), $12 \mu \mathrm{M}$ (lane 7) EhiTRX41 and analyzed by SDS/PAGE. 
Table 3

Templates used for building the structure of EhiUDP-GlcPPase ${ }^{\mathrm{a}}$.

\begin{tabular}{|c|c|c|c|c|c|c|}
\hline PDB code & Expect. & Length & Identities & Positives & Gaps & Resol. ( $(\AA)$ \\
\hline $1 \mathrm{z} 90$ (chain A) & $5.0 \mathrm{E}-098$ & 460 & $192 / 433(44 \%)$ & $284 / 433(65 \%)$ & $9 / 433(2 \%)$ & 1.86 \\
\hline 2i5k (chain A) & 7.0E-098 & 465 & $194 / 445$ (43\%) & $280 / 445(62 \%)$ & $11 / 445(2 \%)$ & 3.10 \\
\hline 2icy (chain A) & 1.0E-096 & 456 & $191 / 420(45 \%)$ & $277 / 420(65 \%)$ & $9 / 420(2 \%)$ & 1.64 \\
\hline 2icx (chain B) & 5.0E-094 & 455 & $188 / 419(44 \%)$ & $274 / 419(65 \%)$ & $11 / 419(2 \%)$ & 1.85 \\
\hline 2oeg (chain A) & $9.0 \mathrm{E}-057$ & 482 & $133 / 403(33 \%)$ & $214 / 403$ (53\%) & $32 / 403(7 \%)$ & 2.30 \\
\hline
\end{tabular}

a 1z90: UDP-GlcPPase encoded by the Arabidopsis thaliana gene At3g03250, APO form [36]; 2i5k: UDP-GlcPPase from Saccharomyces cerevisiae [37]; 2icy: UDP-GlcPPase from Arabidopsis thaliana with bound UDP-Glc [36]; 2icx: UDP-GlcPPase from Arabidopsis thaliana with bound UTP [36]; 2oeg: UDP-GlcPPase from Leishmania major [5].

verify3D score (181) showed that our best model was in between the range of good predicted structures (for 481 residues, minimum score: 102, maximal score: 220 ). The Procheck stereochemical test reported: $0.9 \%$ of residues in the disallowed area of Ramachandran plot [38] indicating that the backbone is folded in a reliable way. Fig. 1 supplementary data shows the final alignment used for building our target. In order to compare secondary structure elements between experimental data and those from modeling, it was included the secondary structure of the APO-form of the enzyme from $A$. thaliana (1z90, see Table 3 ) at the top, and the EhiUDP-GlcPPase model at the bottom of the alignment. Arrows and curly ribbons, respectively, indicate regions predicted as $\beta$-sheet and $\alpha$-helix structures (Fig. 1 Supplementary data).

Fig. 6 shows the best molecular model obtained for E. histolytica UDP-GlcPPase. In the model, $\beta$-sheets and $\alpha$-helices are drawn as violet arrows and red springs, respectively. In cyan are colored the residues of the nucleotide binding site, which are conserved between all known structures. In sticks is depicted the UTP molecule, colored using CPK convention (oxygen: red, carbon: gray, nitrogen: blue and sulfur: yellow). In the model of Fig. 6 of relevance are residues Cys-108, Cys-378 and Met-106, which are represented as van der Waalls spheres, and colored using the same convention as UTP. Met-106 is located in the conserved sequence: Lys-Leu-Asn-Gly-Gly-Leu-Gly-Thr-Xaa-Met-Gly-(Xaa) 4 -Lys, previously reported as the nucleotide binding site for UDP-GlcPPases [5,7]. Cys-108 is also part of the conserved sequence, and it is conserved in the domains aligned in Fig. 1 supplementary data.

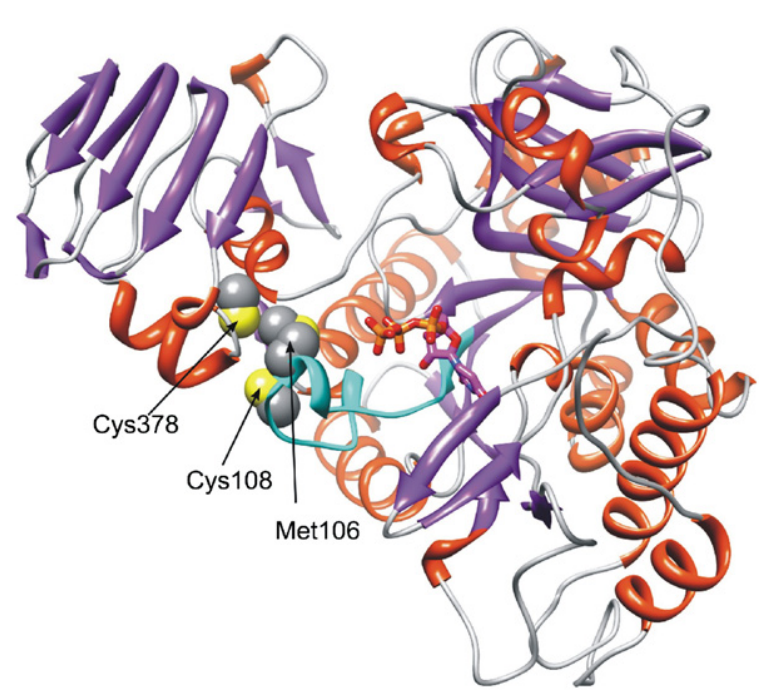

Fig. 6. Molecular modelling of EhiUDP-GlcPPase. The 3-D structure of the amoebic enzyme was modeled using as templates three different UDP-GlcPPases from eukaryotes of known crystallographic structure ( $\alpha$-helices in red, $\beta$-sheet in violet and loops in white). Sticks depict the UTP molecule. Residues C108, C378 and M106 are shown as van der Waalls spheres and sulphur atoms are in yellow. (For interpretation of the references to colour in this figure legend, the reader is referred to the web version of this article).
Concerning Cys-378, it is less conserved, being replaced by serine in three of the six aligned sequences (Fig. 1 Supplementary data). If all the five cysteine residues (Cys-94, Cys-108, Cys-191, Cys-354, and Cys-378) found in EhiUDP-GlcPPase are analyzed by pairs, their respective relative distances are between $\sim 27-33 \AA$, except for the pair Cys-108 and Cys-378, which are $7.43 \AA$ A for each other. The latter distance is longer than $2.03 \AA$, which is a typical distance for an $\mathrm{S}-\mathrm{S}$ bond [39], but it is possible that these regions of the molecule can found closer in the 3-D structure as a consequence of the polypeptides natural global movements [40]. The latter is in good agreement with data in Fig. 5, showing a higher electrophoretic motility or the oxidized enzyme, which would be associated with a more compact structure. Thus, from the model depicted in Fig. 6, it is hypothesized that Cys-108 and Cys-378, surrounding the Met106 residue could be involved in the formation of a disulphide bridge; this provoking the above described changes in activity and motility in SDS/PAGE undergone by the entamoebic enzyme after treatment with the different redox agents.

\subsection{Characterization of methionyl and cysteinyl mutant enzymes}

To further explore on the molecular mechanism responsible for a redox regulation of EhiUDP-GlcPPase and to probe the validity of the prediction derived from molecular modeling (Fig. 6), we constructed different enzymes mutated in their cysteine and methionine residues. On the basis of the information obtained from the 3D model, we constructed mutant enzymes C108S, C378S and C108/ 378S, as well as M106S and M106C. For a more exhaustive study, we also mutated the three additional cysteines found in the entamoebic enzyme constructing mutants C94S, C191S, and C354S. Mutant enzymes were over expressed in E. coli BL21 (DE3) and purified to near $95 \%$ homogeneity by experimental procedures identical to those described for the wild type EhiUDP-GlcPPase. C108S and C378S mutant enzymes were fully active, while the specific activity reached by the double mutant C108/378S was 2 -fold lower than the wild type enzyme. Conversely, M106S was inactive, whereas M106C exhibited a $V_{\max }$ of only $4 \mathrm{U} / \mathrm{mg}$, this being $5 \%$ of the wild type enzyme. These results support the molecular model depicted by Fig. 6, including the essential role of the S-moiety of the methionine residue located in the nucleotide binding site of the enzyme as well as the 3-D architecture arranged by the functional domain [5,7]. On the other hand, C94S, C191S and C354S mutant enzymes exhibited activity levels comparable to the wild type protein.

Interestingly, C108S and C378S single and double mutant EhiUDP-GlcPPases exhibited reduced sensitivity to sulfhydryl oxidants. As shown in Fig. 7, treatments with diamide or SNP causing loss of $90 \%$ activity in the wild type enzyme only inhibited by $\sim 55 \%, \sim 45 \%$ or $20 \%$ to, respectively, the C108S, C378S, or C108/ 3785 mutant enzymes. A similar result was observed for the oxidation with hydrogen peroxide, as C108S and the double mutant enzymes were practically insensitive to the oxidant (Fig. 7). On the other hand, the C94S, C191S, and C354S mutant enzymes behaved 


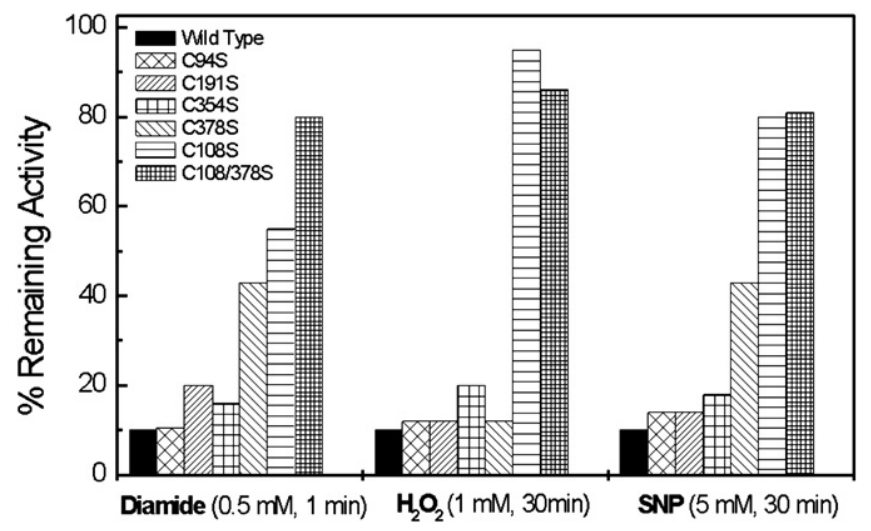

Fig. 7. Response to oxidation by the wild type and mutants in cysteine of EhiUDPGlcPPase. The wild type recombinant UDP-GlcPPase and the different mutant enzymes (as indicated) were incubated at $\mathrm{pH} 8.0$ and $25^{\circ} \mathrm{C}$ with the specified oxidizing agents. After the specified times, samples were assayed for activity in the UDP-Glc synthesis direction.

as the wild type protein when faced with any of the three oxidative treatments (Fig. 7), and all the oxidized mutant enzymes were reverted from inactivation by incubation with the reducing agents. These results strongly support the correctness of the molecular model depicted by Fig. 6, and its prediction concerning the amino acid residues involved in redox regulation, as the absence of at least one of the cysteine residue abolish the possibility of generate the disulphide. However, the C378S mutant UDP-GlcPPase showed no difference to the oxidation by hydrogen peroxide respect to the wild type enzyme (Fig. 7). An explanation for this unexpected behaviour could be attributed to the fact that hydrogen peroxide is able to over-oxidize cysteine or even methionine residues [41]. Thus, in the C378S mutant enzyme the oxidant could be modifying Cys-108 and/or Met-106 in a different way than in the wild type enzyme. Additional support for the specificity of the cysteinyl residues involved in the redox regulation was obtained from results showing differential motility of reduced and oxidized forms of the mutant enzymes in SDS/PAGE. As shown in Fig. 8, mutants C94S, C191S, and C354S and the methionine mutants M106S and M106C exhibited changes in motility depending on the oxidized or reduced states similar to the wild type enzyme. The latter was not observed for single or double mutants in residues Cys-108 and Cys-378, which can be clearly related with the abolishment of the possibility of generate a disulphide linkage in these mutant proteins.

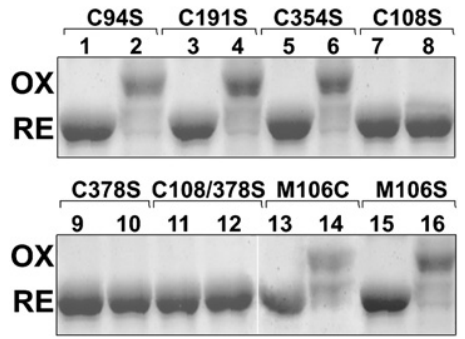

Fig. 8. Analysis of oxidized and reduced EhiUDP-GlcPPase mutants by SDS/PAGE Previous to electrophoresis the purified recombinant mutant enzymes were reduced by 5 min treatment with $1 \mathrm{mM}$ DTT C94S (lane 1), C191S (lane 3), C354S (lane 5), C108S (lane 7), C378S (lane 9), C108/378S (lane 11), M106C (lane 13) and M106S (lane 15). The purified mutants enzymes were instead oxidized by 2 min treatment with $0.5 \mathrm{mM}$ diamide C94S (lane 2), C191S (lane 4), C354S (lane 6), C108S (lane 8), C378S (lane 10), C108/378S (lane 12), M106C (lane 14) and M106S (lane 16).

\section{Discussion}

In the present study, a functionally active UDP-GlcPPase from E. histolytica has been cloned and biochemically characterized, mainly respect to uncover a possible redox regulation of the enzyme. We cloned a nucleotide sequence encoding an UTP-glucose-1phosphate uridylyltransferase (ehiugp), which was obtained from the E. histolytica database. The identity of the amplified (1,446 bp) gene was confirmed by DNA sequencing. The amino acid sequence deduced from ehiugp (481 amino acid residues) showed a high degree of identity with other protozoan UDP-GlcPPases. The size exclusion chromatogram indicates that the active enzyme is a monomer, in agreement with previous finding reported on the L. major enzyme [7]. Also concordant with previous reports were hyperbolic kinetics obtained for Glc1P and PPi, and sigmoidal kinetics for the substrates UTP and UDP-Glc; data resulting characteristic for these kinds of enzymes. Different to that found for UDPGlcPPases from bacteria [6], the entamoebic enzyme showed no activity with other nucleotides: ATP, GTP or TTP.

Numerous works have been reported for other pyrophosphorylases, mainly for ADP-GlcPPases, showing that they are allosterically regulated by intermediates of the major carbon assimilatory pathway in the respective organism [26]. In addition, the ADPGlcPPase from potato tuber was found to be postranslationally regulated by redox modification of specific cysteine residues [42]. It has been proposed that ADP-GlcPPase is the only regulatory enzyme between pyrophosphorylases, in agreement with the protein structure showing that the former is a bigger protein having a characteristic C-term domain [26,43]. Regulatory properties/ mechanisms were never found for any UDP-GlcPPase. Interestingly, the recombinant EhiUDP-GlcPPase was inactivated by incubation with thiol oxidant agents, including diamide, hydrogen peroxide and SNP. The latter inactivation can be completely reverted by chemical (DTT) and biological (L-cysteine, TXN, and TRX) reductants. Outstandingly, our study strongly supports that in E. histolytica the enzyme catalyzing synthesis of UDP-Glc, a first step in oligo and polysaccharides metabolism, may be a target for redox regulation. To the best of our knowledge, our results are novel, not only with respect to the properties of this enzyme but also (and remarkably) concerning the occurrence of this type of regulation in protozoa.

Redox modulation of the EhiUDP-GlcPPase activity seems to be mediated by a mechanism involving oxidative and reductive agents normally found in vivo. Thus, hydrogen peroxide and nitric oxide have been identified as key redox metabolites involved in different intracellular signaling under physiological and stress conditions $[31,32,41,44]$. Action mechanisms for both redox agents include oxidation and S-nitrosylation of proteins $[31,44]$. Thus, in E. histolytica these oxidants could be involved in modifying specific proteins, being UDP-GlcPPase one of the targets, with the enzyme undergoing loss of activity after modification. In the molecular approach studied in the present work, hydrogen peroxide and SNP provoke the formation of a disulphide bond between Cys-108 and Cys-378, both residues being located closer in the 3-D structure and arranging a critical nucleotide binding site together with Met-106 $[5,7]$. The formation of the intramolecular disulphide bridge could partially block substrate access to the active site thus reducing the enzymatic activity. Accordingly, the covalent binding between both cysteine residues seems to produce a conformational change to a more compact molecule that migrates distinct in SDS/PAGE, as reported for other proteins [30,31].

The physiological relevance of the redox modification of EhiUDP-GlcPPase is not limited to the oxidative inactivation but, remarkably, low molecular weight metabolites and redox proteins can reduce the oxidized enzyme with complete recovery of the 
activity. In reverting the disulphide bridge again to the two cysteine residues, they were active TXN and TRX found in trypanosomatids $[18,45,46]$, as well as TRXs from E. histolytica and L-cysteine, a compound that has been identified as a key redox metabolite in the parasite [33]. Our results give additional relevance to the functionality of the TRX system recently identified as a main component of the redox machinery in the protozoon $[16,17]$. In such a way, in E. histolytica TRXs could be involved not only in degradation of reactive oxygen species, but mainly participating in mechanisms for regulating the activity of enzymes through posttranslational modification. The latter has been demonstrated to be a main function played by TRXs in other organisms, including mammals and higher plants [47]. Results reported in the present work strongly support that the metabolism of carbohydrates utilizing UDP-Glc could be strictly controlled with a dependence on the levels of redox conditions in the intracellular environment of the parasite. This would be relevant for the synthesis of different saccharides that play critical roles for the microorganism survival and pathogenesis. It is tempting to speculate, for extending the view, that such a redox mechanism could be also involved in the regulation of other metabolic pathways in E. histolytica and other protozoa.

\section{Conclusions}

We present the molecular cloning, recombinant expression and characterization of the kinetic, regulatory and structural properties of UDP-GlcPPase from E. histolytica. The purified enzyme was sensitive to different oxidant reagents (including diamide, hydrogen peroxide and SNP), which produced a time dependent loss of activity. Interestingly, reducing agents such as DTT, TRX and TXN were able to revert the process by exerting complete recovery of the enzyme activity. Using molecular modelling and sitedirected mutagenesis, Cys-108, Cys-378, and Met-106 were identified as key residues arranging in a 3-D architecture that is critical for activity and redox regulation of the entamoebic enzyme. Results suggest that UDP-GlcPPase could be regulated by redox posttranslational modification in E. histolytica. A rationale view of the regulation would suppose that under oxidant redox environments the usage of Glc-1P to synthesize oligo- and polysaccharides is inhibited and thus the sugar could be derived to catabolic pathways generating reducing power (e.g., NADPH). The latter would reverse the redox scenario, after which reductive conditions could favor the reactivation of UDP-GlcPPase and routes utilizing the sugar nucleotide. Besides the relevance that the latter could have for the parasite carbohydrate metabolism, to the best of our knowledge this is the first report uncovering the occurrence of such a regulatory mechanism in protozoa.

\section{Acknowledgments}

This work was supported by grants from ANCyT (PICT-2007 00668), CONICET (PIP 112-2008-01-02519), and UNL (CAI+D 2009 Orientado \& Redes). LIM and CVP are Fellows from CONICET; and DER, SAG and AAI are investigator career members from the same institution. AAI is recipient of a Fellowship from The John Simon Guggenheim Memorial Foundation.

\section{Appendix. Supplementary data}

Supplementary data associated with this article can be found in the online version, at doi:10.1016/j.biochi.2010.09.019.

\section{References}

[1] W. Stauffer, J.I. Ravdin, Entamoeba histolytica: an update, Curr. Opin. Infect. Dis. 16 (2003) 479-485.

[2] L. Mendonca-Previato, A.R. Todeschini, N. Heise, J.O. Previato, Protozoan parasite-specific carbohydrate structures, Curr. Opin. Struct. Biol. 15 (2005) 499-505.

[3] R. Arya, A. Mehra, S. Bhattacharya, R.A. Vishwakarma, A. Bhattacharya, Biosynthesis of Entamoeba histolytica proteophosphoglycan in vitro, Mol. Biochem. Parasitol. 126 (2003) 1-8.

[4] H.M. Holden, I. Rayment, J.B. Thoden, Structure and function of enzymes of the Leloir pathway for galactose metabolism, J. Biol. Chem. 278 (2003) 43885-43888.

[5] T. Steiner, A.C. Lamerz, P. Hess, C. Breithaupt, S. Krapp, G. Bourenkov, R. Huber, R. Gerardy-Schahn, U. Jacob, Open and closed structures of the UDP-glucose pyrophosphorylase from Leishmania major, J. Biol. Chem. 282 (2007) 13003-13010.

[6] M.B. Bosco, M. Machtey, A.A. Iglesias, M. Aleanzi, UDPglucose pyrophosphorylase from Xanthomonas spp. Characterization of the enzyme kinetics, structure and inactivation related to oligomeric dissociation, Biochimie 91 (2009) 204-213.

[7] A.C. Lamerz, T. Haselhorst, A.K. Bergfeld, M. von Itzstein, R. Gerardy-Schahn, Molecular cloning of the Leishmania major UDP-glucose pyrophosphorylase, functional characterization, and ligand binding analyses using NMR spectroscopy, J. Biol. Chem. 281 (2006) 16314-16322.

[8] F. Martz, M. Wilczynska, L.A. Kleczkowski, Oligomerization status, with the monomer as active species, defines catalytic efficiency of UDP-glucose pyrophosphorylase, Biochem J. 367 (2002) 295-300.

[9] T. Maniatis, E.F. Fritsch, J. Sambrook, Molecular Cloning: A Laboratory Manual. Cold Spring Harbor Laboratory Press, Cold Spring Harbor, NY, 1989.

[10] M. Bradford, A rapid and sensitive method for the quantitation of microgram quantities of protein utilizing the principle of protein-dye binding, Anal. Biochem. 72 (1976) 248-254.

[11] U.K. Laemmli, Cleavage of structural proteins during the assembly of the head of bacteriophage T4, Nature 227 (1970) 680-685.

[12] J. Vaitukaitis, J.B. Robbins, E. Nieschlag, G.T. Ross, A method for producing specific antisera with small doses of immunogen, J. Clin. Endocrinol. Metab. 33 (1971) 988-991.

[13] M.K. Morell, M. Bloom, V. Knowles, J. Preiss, Subunit structure of spinach leaf ADPglucose pyrophosphorylase, Plant Physiol. 85 (1987) 182-187.

[14] C. Fusari, A.M. Demonte, C.M. Figueroa, M. Aleanzi, A.A. Iglesias, A colorimetric method for the assay of ADP-glucose pyrophosphorylase, Anal. Biochem. 352 (2006) 145-147.

[15] S. Aleryani, E. Milo, P. Kostka, Formation of peroxynitrite during thiol-mediated reduction of sodium nitroprusside, Biochim. Biophys. Acta 1472 (1999) 181-190.

[16] D.G. Arias, P.G. Carranza, H.D. Lujan, A.A. Iglesias, S.A. Guerrero, Immunolocalization and enzymatic functional characterization of the thioredoxin system in Entamoeba histolytica, Free Radic. Biol. Med. 45 (2008) 32-39.

[17] D.G. Arias, C.E. Gutierrez, A.A. Iglesias, S.A. Guerrero, Thioredoxin-linked metabolism in Entamoeba histolytica, Free Radic. Biol. Med. 42 (2007) 1496-1505.

[18] N. Reckenfelderbaumer, H. Ludemann, H. Schmidt, D. Steverding, R.L. KrauthSiegel, Identification and functional characterization of thioredoxin from Trypanosoma brucei brucei, J. Biol. Chem. 275 (2000) 7547-7552.

[19] S.R. Wilkinson, D.J. Meyer, J.M. Kelly, Biochemical characterization of a trypanosome enzyme with glutathione-dependent peroxidase activity, Biochem. J. 352 (Pt 3) (2000) 755-761.

[20] K. Arnold, L. Bordoli, J. Kopp, T. Schwede, The SWISS-MODEL workspace: a web-based environment for protein structure homology modelling, Bioinformatics 22 (2006) 195-201.

[21] R.B. Russell, G.J. Barton, Multiple protein sequence alignment from tertiary structure comparison: assignment of global and residue confidence levels, Proteins 14 (1992) 309-323.

[22] J.D. Thompson, D.G. Higgins, T.J. Gibson, CLUSTAL W: improving the sensitivity of progressive multiple sequence alignment through sequence weighting, position-specific gap penalties and weight matrix choice, Nucleic Acids Res. 22 (1994) 4673-4680.

[23] A. Sali, T.L. Blundell, Comparative protein modelling by satisfaction of spatial restraints, J. Mol. Biol. 234 (1993) 779-815.

[24] R. Luthy, J.U. Bowie, D. Eisenberg, Assessment of protein models with threedimensional profiles, Nature 356 (1992) 83-85.

[25] S.K. Gupta, J.R. Sowokinos, I.S. Hahn, Regulation of UDP-glucose pyrophosphorylase isozyme UGP5 associated with cold-sweetening resistance in potatoes, J. Plant Physiol. 165 (2008) 679-690.

[26] M.A. Ballicora, A.A. Iglesias, J. Preiss, ADP-glucose pyrophosphorylase, a regulatory enzyme for bacterial glycogen synthesis, Microbiol. Mol. Biol. Rev. 67 (2003) 213-225.

[27] L.A. Kleczkowski, M. Geisler, I. Ciereszko, H. Johansson, UDP-glucose pyrophosphorylase. An old protein with new tricks, Plant Physiol. 134 (2004) 912-918.

[28] M. Meng, M. Wilczynska, L.A. Kleczkowski, Molecular and kinetic characterization of two UDP-glucose pyrophosphorylases, products of distinct genes, from Arabidopsis, Biochim. Biophys. Acta 1784 (2008) 967-972. 
[29] M. Meng, E. Fitzek, A. Gajowniczek, M. Wilczynska, L.A. Kleczkowski, Domainspecific determinants of catalysis/substrate binding and the oligomerization status of barley UDP-glucose pyrophosphorylase, Biochim. Biophys. Acta 1794 (2009) 1734-1742.

[30] P.A. Lobelle-Rich, R.E. Reeves, Separation and characterization of two UTPutilizing hexose phosphate uridylyltransferases from Entamoeba histolytica, Mol. Biochem. Parasitol. 7 (1983) 173-182.

[31] D.T. Hess, A. Matsumoto, S.O. Kim, H.E. Marshall, J.S. Stamler, Protein S-nitrosylation: purview and parameters, Nat. Rev. Mol. Cell. Biol. 6 (2005) 150-166.

[32] S.G. Rhee, Cell signaling. $\mathrm{H}_{2} \mathrm{O}_{2}$, a necessary evil for cell signaling, Science 312 (2006) 1882-1883.

[33] J. McLaughlin, S. Aley, The biochemistry and functional morphology of the Entamoeba, J. Protozool. 32 (1985) 221-240.

[34] J.G. Kang, M.S. Paget, Y.J. Seok, M.Y. Hahn, J.B. Bae, J.S. Hahn, C. Kleanthous, M.J. Buttner, J.H. Roe, RsrA, an anti-sigma factor regulated by redox change, Embo J. 18 (1999) 4292-4298.

[35] H. Loferer, M. Wunderlich, H. Hennecke, R. Glockshuber, A bacterial thioredoxin-like protein that is exposed to the periplasm has redox properties comparable with those of cytoplasmic thioredoxins, J. Biol. Chem. 270 (1995) $26178-26183$

[36] J.G. McCoy, E. Bitto, C.A. Bingman, G.E. Wesenberg, R.M. Bannen, D.A. Kondrashov, G.N. Phillips Jr., Structure and dynamics of UDP-glucose pyrophosphorylase from Arabidopsis thaliana with bound UDP-glucose and UTP, J. Mol. Biol. 366 (2007) 830-841.

[37] A. Roeben, J.M. Plitzko, R. Korner, U.M. Bottcher, K. Siegers, M. Hayer-Hartl, A. Bracher, Structural basis for subunit assembly in UDP-glucose pyrophosphorylase from Saccharomyces cerevisiae, J. Mol. Biol. 364 (2006) 551-560.
[38] G.N. Ramachandran, C. Ramakrishnan, V. Sasisekharan, Stereochemistry of polypeptide chain configurations, J. Mol. Biol. 7 (1963) 95-99.

[39] R.A. Laskowski, M.W. MacArthur, D.S, Moss, JM. Thornton, PROCHECK: a program to check the stereochemical quality of protein structures, J. Appl. Crystallogr. 26 (1993) 283-291.

[40] M. Gerstein, A.M. Lesk, C. Chothia, Structural mechanisms for domain movements in proteins, Biochemistry 33 (1994) 6739-6749.

[41] S.G. Rhee, S.W. Kang, W. Jeong, T.S. Chang, K.S. Yang, H.A. Woo, Intracellular messenger function of hydrogen peroxide and its regulation by peroxiredoxins, Curr. Opin. Cell. Biol. 17 (2005) 183-189.

[42] Y. Fu, M.A. Ballicora, J.F. Leykam, J. Preiss, Mechanism of reductive activation of potato tuber ADP-glucose pyrophosphorylase, J. Biol. Chem. 273 (1998) $25045-25052$

[43] M.A. Ballicora, E.D. Erben, T. Yazaki, A.L. Bertolo, A.M. Demonte, J.R. Schmidt, M. Aleanzi, C.M. Bejar, C.M. Figueroa, C.M. Fusari, A.A. Iglesias, J. Preiss, Identification of regions critically affecting kinetics and allosteric regulation of the Escherichia coli ADP-glucose pyrophosphorylase by modeling and pentapeptide-scanning mutagenesis, J. Bacteriol. 189 (2007) 5325-5333.

[44] M.W. Foster, D.T. Hess, J.S. Stamler, Protein S-nitrosylation in health and disease: a current perspective, Trends Mol. Med. 15 (2009) 391-404.

[45] S.A. Guerrero, M. Montemartini, R. Spallek, H.J. Hecht, P. Steinert, L. Flohe, M. Singh, Cloning and expression of tryparedoxin I from Crithidia fasciculata, Biofactors 11 (2000) 67-69.

[46] C.V. Piattoni, V.S. Blancato, H. Miglietta, A.A. Iglesias, S.A. Guerrero, On the occurrence of thioredoxin in Trypanosoma cruzi, Acta Trop. 97 (2006) 151-160

[47] Y. Meyer, B.B. Buchanan, F. Vignols, J.P. Reichheld, Thioredoxins and glutaredoxins: unifying elements in redox biology, Annu. Rev. Genet. 43 (2009) 335-367. 